\title{
IMPACTO DE LAS CRIPTOMONEDAS EN LA POBLACIÓN ESTUDIANTIL UNIVERSITARIA DE LA REPÚBLICA DE PANAMÁ
}
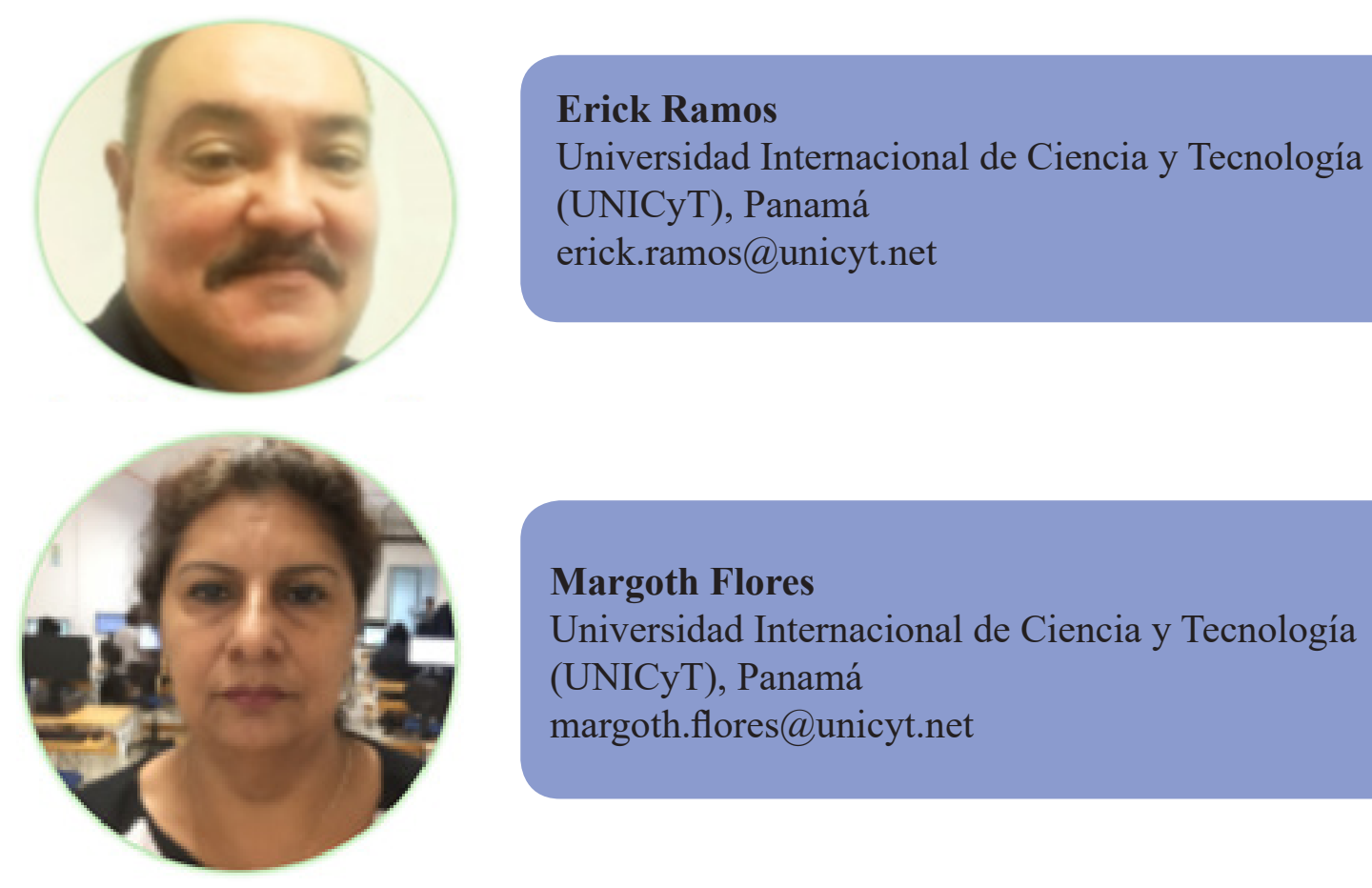

\section{Margoth Flores}

Universidad Internacional de Ciencia y Tecnología (UNICyT), Panamá margoth.flores@unicyt.net

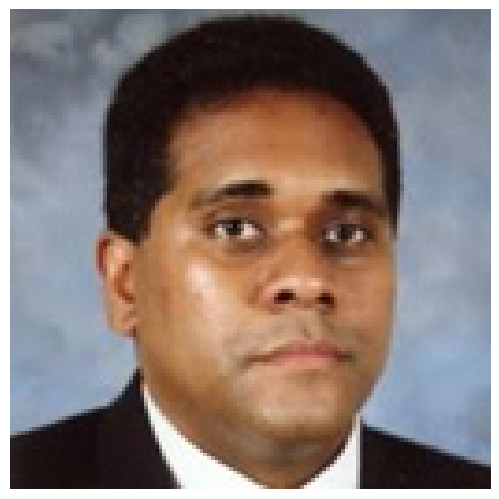

\section{Ricardo Zeballos \\ Universidad Internacional de Ciencia y Tecnología (UNICyT), Panamá ricardo.zeballos@unicyt.net}

\section{RESUMEN}

Una nueva innovación disruptiva aparece en el horizonte financiero internacional y de la República de Panamá, las criptomonedas constituyen un medio digital de intercambio de uso en varios mercados con características similares a lo que ocurre con los productos, mercancías o materias primas llamados "Commodities" en los mercados internacionales y de forma similar a los que ocurre con el oro. Aun cuando cada vez más se incrementa su volumen transaccional, el valor de las criptomonedas es muy volátil y se puede aseverar que no 
existe una guía especifica en las Normas Internacionales de Información Financiera (NIIF) sobre el tratamiento contable de las criptomonedas a pesar de lo acelerado de su uso, dejando sin respuesta la pregunta: ¿Cómo se podrán contabilizar? El aspecto de interés de esta investigación se focaliza en analizar el grado de conocimiento y aceptación de las criptomonedas en la población estudiantil universitaria en la República de Panamá. Panamá es hoy en día uno de los centros económicos más importantes, tanto de la región de Centro América como de Latinoamérica y el Caribe. Por otra parte, está demostrado que el uso de la economía digital para las remesas en lugar de los pagos en efectivo es beneficioso para las personas de bajos recursos en países en vías de desarrollo y Panamá cuanta con una legislación que prevé el uso de activos digitales. Esta investigación pretende brindar luces sobre un fenómeno que puede convertirse en el nuevo y mejor uso de Internet. La metodología utilizada en el desarrollo de esta investigación es una metodología particular, basada en el uso de técnicas de investigación propias tanto de la investigación documental como de la investigación descriptiva y la investigación de campo. Aunque esta es una investigación en desarrollo, sus resultados parciales demuestran que la seguridad de las criptomonedas está basada en un servicio de valor agregado conocido como "Blockchain". Algunas de sus cualidades más importantes son: el hecho de Imposibilitar que un tercero determine quien, cuando y el monto de una transacción hecha por un usuario. En tal sentido los autores consideran que ciertamente las criptomonedas son una innovación disruptiva que plantea nuevos retos a la banca internacional, a la Banca Panameña y al público en general. También se considera que, aunque la seguridad de las criptomonedas es buena, nunca será mayor que la de los sistemas informáticos en general.

Palabras clave: Estudiantes universitarios de la República de Panamá, Criptomonedas, Criptovalores, Seguridad, Brecha digital, Blockchain, Innovación disruptiva. 
Impacto de las criptomonedas en la población estudiantil universitaria de la República de Panamá

Erick Ramos, Margoth Flores y Ricardo Zeballos. (10:27)

\title{
IMPACT OF THE CRYPTOMONDS IN THE UNIVERSITY STUDENT POPULATION OF THE REPUBLIC OF PANAMA
}

\begin{abstract}
A new disruptive innovation appears in the international financial horizon and in the Republic of Panama, cryptocurrencies are a digital means of exchange for use in several markets with characteristics similar to what happens with products, merchandise or raw materials. called "Commodities" in international markets and similar to what happens with gold. Even when its transaction volume increases more and more, the value of cryptocurrencies is very volatile and it can be asserted that there is no specific guide in the International Financial Reporting Standards (IFRS) on the accounting treatment of cryptocurrencies despite the accelerated of its use, leaving the question unanswered: ¿How it can be counted? The interesting aspect of this research is focused on analyzing the degree of knowledge and acceptance of cryptocurrencies in the university student population in the Republic of Panama. Panama is today one of the most important economic centers, both in the Central American region and in Latin America and the Caribbean. On the other hand, it has been demonstrated that the use of the digital economy for remittances instead of cash payments is beneficial for low-income people in developing countries and Panama as long as it has legislation that provides for the use of digital assets. This research aims to shed light on a phenomenon that can become the new and better use of the Internet. The methodology used in the development of this research is a particular methodology, based on the use of research techniques of all tree documentary research, descriptive research and field research. Although this is a research in development, its partial results show that the security of cryptocurrencies is based on a value-added service known as "Blockchain". Some of its most important qualities are: the fact that it is impossible for a third party to determine who, when and the amount of a transaction made by a user. In this regard, the authors believe that cryptocurrencies are a disruptive innovation that poses new challenges to international banking, the Panamanian Banking and the general public. It is also considered that, although the security of cryptocurrencies is good, it will never be greater than that of computer systems in general.
\end{abstract}

Keywords: University students from the Republic of Panamá, Cryptocurrencies, Crypto values, Security, Digital gap, Blockchain, Disruptive innovation. 


\section{INTRODUCCIÓN}

Un fenómeno cada vez más recurrente es la aparición de tecnologías o servicios de valor agregado que irrumpen en el quehacer cotidiano y transforman la manera en la que se hacen las cosas, muchas veces los usuarios comienzan a hacer uso de un producto o servicio que antes no utilizaban y esto provoca tanto un importante cambio en la forma de hacer las cosas como en la manera de pensar de la sociedad, por ello se les conoce como tecnologías disruptivas o innovación disruptiva.

Si bien es cierto que Internet a contribuido significativamente a elevar la calidad de vida de la sociedad contemporánea, permitiendo generar riqueza, esta no se ha distribuido equitativamente entre la sociedad como o demuestran los niveles de pobreza, sobre todo en los países en vías de desarrollo y el fenómeno de la" Brecha Digital". La inequidad parce florecer alrededor del mundo.

Sin embargo, la única propuesta seria para resolver estos problemas de la humanidad parecen ser la redistribución de la riqueza de forma más equitativa, sin que la misma cuente aun con un método o una política que la lleve a la práctica, más allá de la democracia.

Por otro lado, las finanzas han evolucionado hasta un modelo funcional centralizado, caracterizado por la intermediación bancaria, donde las operaciones son controladas generalmente por un banco central que emite y asegura las operaciones con monedas reconocidas. No obstante, esta intermediación supone unos costos que oscilan entre un $7 \%$ y un $20 \%$ en muchos casos y un tiempo de demora que en promedio se calcula en 3 días.

Las criptomonedas o criptodivisas podrían no solo reducir el tiempo de demora de las transacciones, sino también terminar con la centralización de las transacciones, pre-distribuyendo la riqueza e incluso democratizándola al permitir que cada individuo u organización reciba y administre directamente sus riquezas y productos.

Entonces la pregunta que surge es: ¿Cuan preparado se está para afrontar la disrupción? Las criptomonedas se presentan como una de las más importantes innovaciones disruptivas de las finanzas contemporáneas. Es por ello por lo que esta investigación se propone el objetivo general de analizar el grado de conocimiento y aceptación de las criptomonedas en la población estudiantil universitaria en la República de Panamá.

En el mismo orden de ideas, los objetivos específicos incluyen: Identificar lo último en tecnología de las criptomonedas a nivel mundial y a nivel local; Conocer la penetración (conocimiento y aceptación) de las criptomonedas en la población estudiantil en la República de Panamá; Generar un análisis que permita cuantificar el conocimiento y la aceptación de las criptomonedas en la población estudiantil universitaria en la 
Impacto de las criptomonedas en la población estudiantil universitaria de la República de Panamá

Erick Ramos, Margoth Flores y Ricardo Zeballos. (10:27)

República de Panamá; Desarrollar un documento para publicar los resultados, conclusiones y recomendaciones de esta investigación.

\section{CRIPTOMONEDAS}

Probablemente una de las actividades más antiguas de la humanidad es la economía, formalizada tal vez a partir de la civilización en Mesopotamia cuatro mil años antes de cristo (4000 A.C.), aunque su aparición es anterior a la era prehistórica unos nueve mil años antes de cristo (9000 A.C.).

Es así como, de acuerdo con el Diccionario de la Lengua Española, La Economía se puede definir como: [1] "Conjunto de bienes y actividades que integran la riqueza de una colectividad o un individuo." O como: "Ciencia que estudia los métodos más eficaces para satisfacer las necesidades humanas materiales, mediante el empleo de bienes escasos." (Diccionario de la Lengua Española, 2017).

En tal sentido, la Economía tiene que ver con la calidad de vida ya que tiene relación con el intercambio de bienes o servicios entre los individuos de la sociedad. Para ello se hace uso del dinero, el que podemos definir, según la edición del tricentenario del Diccionario de la lengua española como el: [2] "medio de cambio o de pago aceptado generalmente" (Diccionario de la lengua española en la Web, 2017).

Los medios de pago más comunes en la economía contemporánea son las monedas, los billetes, las tarjetas de débito, las tarjetas de crédito, las transferencias electrónicas y los cheques.

También se reconoce que, tanto las monedas como los billetes son un conjunto de signos representativos del dinero circulante en cada país, instrumento aceptado como unidad de cuenta, medida de valor y medio de pago. Sin embargo, la dinámica del comercio en la actualidad ha impulsado la necesidad creciente de uso de herramientas de intercambio reconocidas por ambas partes en una transacción.

El dinero plástico, nombre dado a las tarjetas de crédito, a las tarjetas de débito y a otros instrumentos de pago similares, aunque menos comunes, es una solución parcial a la situación de contar con un instrumento de pago aceptado por ambas partes, según la publicación en la web "La Enciclopedia Política", el dinero plástico [3] "es una modalidad de dinero electrónico. Consiste en una tarjeta de plástico, emitida por un banco o una entidad financiera especializada" (Borja, 2017). La entidad financiera se encarga de autorizar al usuario los productos y servicios que adquiere, pero también a obtener efectivo en forma de anticipo, en un cajero automático o ATM.

Es así como, en la actualidad el intercambio de bienes y servicios se lleva a cabo no solamente de forma local, es decir entre los miembros de una misma sociedad (geográficamente cercana) sino 
también entre individuos de distintas partes del mundo, a través del uso de herramientas económicas y la tecnología. Por otra parte, la economía digital es el término utilizado para definir la confluencia de la infraestructura telemática, las actividades económicas y sociales a través de Internet, redes de datos móviles y otros servicios telemáticos de valor agregado.

Según un informe del Grupo de Investigaciones del Banco Mundial sobre el Desarrollo [4] "La integración de los pagos digitales en las economías de las naciones emergentes y en desarrollo aborda cuestiones fundamentales del crecimiento económico generalizado y el empoderamiento financiero de las personas". (Banco Mundial, 2014).

Esta evidenciado que las operaciones de desembolsos digitales brindan prerrogativas a los compradores y a los acreedores en las economías en desarrollo, y que dichos pagos efectivamente aumentan el acceso de los ciudadanos a las herramientas financieras digitales.

Por su parte, la Comisión Económica para América Latina (CEPAL) señala que: [5]” La economía digital es una fuerza crucial para impulsar el cambio estructural, avanzar en la reducción de la desigualdad y fortalecer la inclusión social que tanto necesitan los países de la región." (CEPAL, 2012).

En ese sentido, la economía digital facilita la transformación representativa que coadyuvan la creación de pymes y por ende el crecimiento de la inclusión social, cuestión esta que incumbe a todos los países en vías de desarrollo.

También, información ofrecida por el Administrador de la Autoridad de Innovación Gubernamental, (A.I.G.) el Lic. Irving Halman, en entrevista ofrecida recientemente (08-11'2017) en el canal de TV Televisora Nacional, S.APanamá, quien afirmo que Panamá se prepara para abordar la economía digital y que es necesario acometer esa tarea ya que es un fenómeno mundial donde se pretende abordar al usuario de los servicios financieros de forma directa, sin la clásica intermediación financiera de la banca, Además argumento que esto sucede ya que los usuarios no desean ver atrasadas sus operaciones al tener que alinearse detrás de una fila o esperar los distintos niveles de aprobación de las operaciones digitales convencionales a través de la intermediación bancaria.

En efecto, la Ley 51 del 22 de junio de 2008, Publicada en la Gaceta Oficial № 26090 del 24 de julio del mismo año, establece: [6] "Que define y regula los documentos electrónicos y las firmas electrónicas y la prestación de servicios de almacenamiento tecnológico de documentos y de certificación de firmas electrónicas y adopta otras disposiciones para el desarrollo del comercio electrónico". Pretende preparar a Panamá para el advenimiento de la economía digital. (Órgano Judicial del Gobierno de Panamá, 2008). 
Impacto de las criptomonedas en la población estudiantil universitaria de la República de Panamá Erick Ramos, Margoth Flores y Ricardo Zeballos. (10:27)

En el mismo orden de ideas, uno de los más recientes servicios de valor agregado, incorporado a la actividad económica contemporánea son las criptomonedas. Éstas son: [18]" Un activo que puede subir o bajar", También, según Chohan son [19] "Un instrumento digital de intercambio" de reciente incorporación a la economía de algunos países.

Así mismo, las criptomonedas se presentan como una de las más importantes innovaciones disruptivas de las finanzas contemporáneas. En la actualidad existen más de una decena de estas herramientas diferenciadas por su tecnología y características, sin embargo, casi todas son similares entre sí y la primera de ellas en aparecer llamada Bitcoin.

La historia moderna refleja que la creación de Bitcoins comenzó en 2009 por un individuo identificado por el sobrenombre de Satoshi Nakamoto. [20] En 2016, producto de reseñas en medios de comunicación televisivo y escritos (la BBC y revistas especializadas) se develo que la verdadera identidad de Satoshi es Craig Wright, un empresario australiano experto en criptografía, quien demostró haber creado una intrincada fórmula la cual puede ser procesada a través un computador, creando un "bloque" de datos que contiene Bitcoins. [7] Hay una cantidad máxima de Bitcoins que existirá, y hay rendimientos decrecientes integrados en el sistema. Por ejemplo, los usuarios anteriormente podían crear 50 Bitcoins en un bloque, pero desde finales de 2012, la cantidad creada se reducía a 25 (Matonis, 2013).

La seguridad de esta herramienta informática está basada en un servicio de valor agregado conocido como "Blockchain". Algunas de sus cualidades más importantes son: el hecho de Imposibilitar que un tercero determine quien, cuando y el monto de una transacción hecha por un usuario; Permite a los usuarios comprobar el pago o la identidad del pagador bajo condiciones excepcionales y Permite detener el uso de los medios de pago reportados como robados.

No obstante, La seguridad de muchas otras soluciones tecnológicas tampoco es absoluta, sin embargo, la sociedad hace uso intensivo de ella, aunque la vida misma este en juego; tal es el caso de los sistemas de vuelo de las aeronaves y los de soporte critico a la vida.

En tal sentido, un artículo publicado en la página oficial del FBI, en marzo de 2012, con motivo de reseñar la conferencia de Cyber Seguridad en la ciudad de San Francisco, EE. UU., el ex director del FBI: Robert Mueller III señalo que: [8] "Yo estoy convencido de que solo hay dos tipos de compañías: aquellas que han sido hackeadas y aquellas que lo serán. Y ellas convergen en una sola categoría: compañías que han sido hackeadas y que serán hackeadas nuevamente". (Mueller III, 2012) (traducción propia).

En el mismo orden de ideas, recientemente (07-12-2017), una importante empresa de minería en la nube, relacionada con la criptomoneda Bitcoin reporto un evento de violación de su seguridad. Se trata de NiceHash [21] "un mercado que agrupa a los pequeños mineros, que aportan mínimas 
cantidades de poder de cómputo, confirmo que fue hackeada y los atacantes robaron Bitcoins de las carteras de sus clientes". Según esta y otras reseñas las perdidas suponen unos $\$ 60,000,000.00$

[9] Luego de la aparición de la criptomoneda Bitcoin, han aparecido otras criptomonedas como: Namecoin, Litecoin y Freicoin. Muchas otras criptomonedas han sido creadas, aunque no todas han sido exitosas, en especial aquellas que no han aportado ninguna innovación. En sus casi 8 años de existencia, las criptomonedas han ido ganando paulatinamente la atención del público general y de los medios de comunicación (Surowiecki, 2011).

Desde el año 2011, el interés del público en general ha aumentado rápidamente, en especial, durante el ascenso vertiginoso de Bitcoin en abril de 2013. En Panamá comienza a darse el uso de las criptomonedas como lo destaca un artículo de prensa digital que señala que: [10] "La capital del país registra 19 negocios que admiten el uso de criptomonedas, en particular el bitcóin, como un medio alternativo de pago junto con el dólar y el dinero plástico. La expectativa de uso de estas divisas digitales, estiman sus promotores, se cifra en sobrepasar los 30 comercios cuando finalice el presente año. Significará un incremento de más del 50\% dentro de un fenómeno global con ribetes semejantes a los del inicio de la internet." (Castaño, 2017).

En tal sentido, las criptomonedas ya están en funcionando en el territorio de la República de Panamá y se espera un crecimiento en su uso, aun cuando también expone Castaño que su uso en Panamá es casi exclusivo por parte de extranjeros. En ese orden de ideas Janice Becerra, abogada de la firma Panamá Legal Group, citada por Castaño en su artículo, sostiene que: [11] "el fenómeno ha tardado en despegar entre los panameños. El desconocimiento del sistema o el temor a la novedad disminuye, por ahora, las posibilidades de un activo digital gestionado a través de un sistema de pago gobernado solo por las personas. "Todos nuestros clientes son extranjeros, ninguno de nuestro país. Pero sí hemos visto que el público local empieza a preguntar por el alcance de las criptomonedas, sobre todo del bitcóin” (Becerra, 2017).

En la República de Panamá la banca se construyó de manera gradual, siendo el antecedente más connotado la apertura del International Bank Corporation, en agosto de 1904. Ya en 2015 el sistema financiero de Panamá se consolida como uno de los más importantes de la región, así lo reseña la prensa local: [12] "El sistema financiero nacional se ha posicionado como uno de los más sólidos de la región. Las 91 entidades que conforman la industria bancaria, actualmente, le dan trabajo a, aproximadamente, 20 mil personas, entre nacionales y extranjeros." (Diario La Estrella, 10-05-2015, pp. 1).

También es importante señalar la importancia de las operaciones financieras que se llevan a cabo en el sistema financiero de Panamá, con lo que queda evidenciado en la misma reseña de la prensa nacional que: [12] "El Centro Bancario Internacional, creado en 1970, aporta el 7.5\% del 
Impacto de las criptomonedas en la población estudiantil universitaria de la República de Panamá Erick Ramos, Margoth Flores y Ricardo Zeballos. (10:27)

Producto Interno Bruto (PIB) de Panamá, lo cual convierte a la intermediación financiera en la sexta actividad que más aporta el PIB panameño.” (Diario La Estrella, 10-05-2015, pp. 1).

Así mismo, en otra edición del mismo diario se afirma que; [13] "El Centro Bancario Internacional tiene relevancia en la región, lo cual se muestra en los niveles de competitividad que Panamá mantiene en el Índice Global de Competitividad del Foro Económico Mundial (2014-2015) en el Pilar: Sofisticación del Mercado Financiero, en el cual Panamá se posiciona como el número 22 de 144 países." (Diario La Estrella, 06-05-2015, pp. 1).

Por otro lado, los estudiantes universitarios, y en particular los de la República de Panamá representan el futuro más inmediato del país, su incorporación al mercado laboral en términos temporales representa el poder de decisión de una generación, acostumbrada a las tecnologías disruptivas y con un gran poder de adaptación que facilita la permeabilidad de las nuevas tecnologías y de los servicios de valor agregado que aparecen alrededor de ellas.

\section{PERTINENCIA}

La economía de un país es una de las principales responsabilidades de sus autoridades y en la República de Panamá, esto no es una excepción. Para sus autoridades el contar con una bonanza económica y con relativa estabilidad, a pesar de los diferentes problemas en la región y el mundo, representa una constante causa de mayor trabajo.

En tal sentido, mientras la mayoría de los países vecinos y regionales crecen a una tasa menor al 3\%, Panamá lleva más de 10 años con una tasa mayor al 5\%, con el valor agregado que da el desarrollo de grandes obras de infraestructura como las segundas esclusas del canal de Panamá, obra también conocida como la ampliación del Canal de Panamá, la ampliación del Aeropuerto internacional de Tocumen y las obras adelantadas de las líneas número dos, tres y cuatro del Metro de Panamá. Todas estas obras con profundo impacto en la economía del país y de la región.

De acuerdo con información publicada en el diario local La Prensa, en su versión del domingo 15 de octubre de 2017, [16] "Panamá es uno de los países de la región que menos presupuesto destina para el desarrollo de la ciencia, tecnología e innovación en Latinoamérica. Mientras la media regional es de $0.7 \%$ del producto interno bruto, el país solo destina el 0.2\%." (Diario La Prensa, 15-10-2017).

En un informe del Banco Mundial, previamente mencionado, se afirma que: [4] "el establecimiento de sistemas de pagos digitales para las remesas en lugar de los pagos en efectivo es sumamente beneficioso para las personas pobres de los mercados emergentes y contribuye al desarrollo financiero. “(Banco Mundial, 2014). 
El resultado de esta investigación permitirá a las autoridades de Panamá contar con una fuente confiable de información sobre criptomonedas y su Impacto en la población estudiantil universitaria de la República de Panamá. Esta información le permitirá planificar y generar y políticas públicas dirigidas a regular el uso de las criptomonedas en el país.

Los resultados de esta investigación apoyarán el cumplimiento del Artículo 72 de la Ley 51 del 22 de julio de 2008, por parte de la Dirección General de Comercio Electrónico, al proveer información pertinenteparala formación delos Comités Consultivos, en materia de las criptomonedas.

Panamá es hoy en día uno de los centros económicos más importantes, tanto de la región de Centro América como de Latinoamérica y el Caribe. Un artículo de prensa local en su sitio web que indica que: [12] "El desarrollo del Centro Bancario Internacional de Panamá se ha fortalecido y consolidado como una de las principales de la región latinoamericana", al punto que consideran en la Superintendencia que el Centro está 'llamado a convertirse en el Centro Bancario Internacional de Latinoamérica y Hub Financiero para las Américas'. (Diario La Estrella, 10-05-2015).

Con el cambio climático y los fenómenos atmosféricos castigando los campos de cultivo y de crianza en medio de la globalización, y contando Panamá con dos puertos estratégico (Panamá y Colon) así como el Aeropuerto Internacional Tocumen, es evidente considerar a Panamá como un importante enlace logístico de trascendencia regional e internacional. La presencia de varias instituciones financieras de capital internacional, así como de un buen número de empresas trasnacionales da fe de ello.

En tal sentido, [12] algunas cifras muestran que para el año 2015 en la República de Panamá se contaba con noventa y un (91) bancos con licencia para operar, con un aporte al Producto Interno Bruto (PIB) de Panamá del 7.5\%. alcanzando la sexta posición entre las actividades que aportan al P.I.B. de Panamá. El Centro Bancario de Panamá fue creado en 1970, con la aprobación del Decreto 238 del 2 de julio de 1970. Con 47 años de existencia y la creación en 1998 de la Superintendencia de Bancos de Panamá, como autoridad reguladora de la banca, la madurez queda comprobada. (Diario La Estrella, 10-05-2015).

Por otra parte, los estudiantes universitarios en Panamá no escapan a la realidad de interesarse en las nuevas tecnologías, pero la falta de información oportuna podría ocasionar situaciones que van desde el rechazo prematuro del uso de la tecnología hasta el uso indiscriminado o abusivo de tecnologías cuya seguridad no ha sido comprobada por las autoridades. Los resultados de esta investigación pondrán en las manos de los futuros profesionales la información necesaria para decidir la incorporación o el rechazo de las criptomonedas a la Banca Comercial de Panamá. 
Impacto de las criptomonedas en la población estudiantil universitaria de la República de Panamá Erick Ramos, Margoth Flores y Ricardo Zeballos. (10:27)

En tal sentido, un diagnóstico negativo en cuanto a la comprensión del fenómeno de las criptomonedas por parte de los estudiantes alertaría a las autoridades a diseñar políticas y planes de educación y difusión más apropiados para solventar esa situación.

\section{BENEFICIOS}

Conocer el grado de penetración en la población estudiantil universitaria de la República de Panamá, de la cultura detrás de las criptomonedas permitirá a las autoridades gubernamentales diseñar políticas y planes de desarrollo más ajustados a la realidad económica mundial, protegiendo el patrimonio cultural y económico de los panameños y su importante centro financiero.

Así lo establece Geoffrey Lamb de la Fundación Bill y Melinda Gates, citado en un estudio del Banco Mundial previamente mencionado, cuando afirma que: [4] "los ciudadanos apreciarán y aprenderán rápidamente a utilizar los sistemas de pagos digitales. Pero es necesario que los Gobiernos establezcan la visión, las plataformas digitales y la normativa para que los cientos de millones de personas que actualmente se encuentran excluidas participen plenamente en la economía moderna". (Banco Mundial, 2014).

En un país que constantemente está revisando su legislación para garantizar a la mujer la igualdad de derechos, el reconocimiento y la incorporación de las herramientas de la economía digital coadyuva el logro de este objetivo por parte del estado.

Así lo concluye un estudio del Banco Mundial previamente mencionado cuando establece que: [4] "Los servicios financieros digitales reducen los costos y aumentan la seguridad al enviar, pagar y recibir dinero. La mayor inclusión financiera resultante también es de vital importancia para el empoderamiento de las mujeres". (Banco Mundial, 2014).

Es por ello, por lo que el gobierno de la República de Panamá también podría rechazar la incorporación de las criptomonedas al país al detectarse debilidades de las mismas o amenazas a la economía del país. Algunos gobiernos ya han rechazado el uso de las criptomonedas por temor a su impacto negativo en sus economías.

En ese orden de ideas, en el año 2014, El Banco Central de Bolivia BCB prohibió el uso de las criptomonedas en Bolivia. [17] "El Banco Central de Bolivia (BCB), mediante una resolución de directorio, prohibió el uso en el país de monedas virtuales conocidas como Bitcoins y que tienen adeptos en otros países.” (Diario Página Siete, versión electrónica del 21-06-2014).

También, el Ministerio de Educación de la República de Panamá, (MEDUCA) podrá desarrollar estrategias y planes acordes con el desarrollo de la economía moderna y que incluyan esta pieza importante de la tecnología y economía emergente como lo son las criptomonedas. 
De forma similar, el sector financiero de Panamá podrá prepararse de forma más adecuada y de forma más gradual a la incorporación de las criptomonedas a la cultura económica y financiera de la banca moderna, o tomar otro camino basado en las probables debilidades o amenazas.

Así mismo, los panameños se podrán beneficiar de unas políticas que preserven el eslabón de su economía relativo al centro financiero y garanticen su participación en la actividad económica, sin requerir de mayor mano de obra calificada en el tema.

Por otra parte. Los panameños se beneficiarán de poseer una mano de obra calificada propia y para la exportación, capaz de proteger la economía panameña elevando los niveles de calidad de vida al aumentar el potencial de las fuentes de empleo.

Los hispanohablantes contaran con un instrumento de diagnóstico y soporte, así como una metodología probada para entender mejor el fenómeno de las criptomonedas y sus efectos en la banca de Panamá.

\section{IMPACTO ESPERADO}

Se espera que la información recopilada y el análisis de los datos permita brindar a las autoridades competentes en la República de Panamá un visión realista y actualizada no solo de la penetración de la cultura de las criptomonedas en la población estudiantil universitaria de la República de Panamá, sino también de las recomendaciones y sugerencias que arroje el análisis del equipo de investigadores multidisciplinario, autores de esta investigación.

El análisis y la discusión de los resultados de esta investigación deben impulsar el interés tanto de las autoridades competentes como del público general que es al final de cuentas el más interesado en aclarar las perspectivas de las criptomonedas en la economía digital de Panamá.

En ese orden de idea, el producto de esta investigación pretende coadyuvar a la disminución de la incertidumbre sobre el tema de las criptomonedas y su impacto en los estudiantes universitarios de Panamá.

\section{Materiales y métodos}

La metodología en desarrollo es una metodología particular de esta investigación, basada en el uso de técnicas de investigación propias tanto de la investigación documental como de la investigación descriptiva y la investigación de campo ya que se realizarán encuestas a una muestra de la población estudiantil universitaria de la República de Panamá para conocer el grado de comprensión y el impacto de las criptomonedas en la economía local.

La metodología de esta investigación se ha dividido en cinco (5) faces, una primera fase de investigación descriptiva que está en desarrollo por ser esta fase una que abarca desde el comienzo 
Impacto de las criptomonedas en la población estudiantil universitaria de la República de Panamá Erick Ramos, Margoth Flores y Ricardo Zeballos. (10:27)

hasta el final de la investigación, donde se pretende describir los tres elementos fundamentales de esta investigación: la población estudiantil universitaria de Panamá, por ser esta la generación que deberá asumir la responsabilidad de liderar la transformación y adaptación de la Banca de Panamá y su contexto financiero en los próximos años, las criptomonedas y su contexto de economía digital, y la relación que existe entre ellos.

En esta fase se ha de obtener los datos del número de estudiantes universitarios de la República de Panamá, así como el número de universidades autorizadas y activas en la Republica de Panamá, la distribución de la matrícula en programas de nivel de pregrado y otros datos demográficos que ayudaran a calcular la muestra de la población de esta investigación. Con respecto a este último punto, el equipo de investigadores ha decidido contemplar que la muestra se forme solo con estudiantes de la provincia de Panamá, habida cuenta que aproximadamente el $60 \%$ de la población estudiantil universitaria de pregrado se encuentra matriculada en los respectivos campus en la Ciudad de Panamá.

Así mismo, en esta fase se ha de encontrar datos sobre el estado de la tecnología de las criptomonedas, su nivel de aceptación en la economía internacional, las ventajas y desventajas presentes y la percepción general de los expertos en áreas de interés, como por ejemplo la seguridad informática, las regulaciones legales y otros datos afines.

En una segunda fase se estableció el mecanismo para encontrar el estado actual de la relación entre el fenómeno de las criptomonedas y la población estudiantil universitaria de Panamá, en esta etapa se ha dispuesto hacer uso de una metodología de campo. Como instrumento para recolectar la información de este trabajo de investigación, se ha dispuesto hacer uso de la técnica de la encuesta, que se aplicará a los estudiantes de las universidades de la ciudad de Panamá.

En tal sentido, se ha establecido que el instrumento sea validado mediante la consulta a expertos y luego el mismo será aplicado a una muestra de la población de estudiantes universitarios de pregrado de las universidades con por lo menos una sede en la provincia de Panamá. En tal sentido, la fiabilidad de la muestra de estudiantes de las universidades de Panamá se calculará a través del Modelo Alfa de Cronbach.

Una tercera fase proveerá la consolidación y análisis de los datos obtenidos mediante el uso de técnicas de estadística descriptiva, que permitirán describir las características de la distribución de los puntajes recopilados, tales como: Media, Moda, Mediana, Dispersión, entre otras. El análisis llevara a la generación de resultados, conclusiones y recomendaciones por parte del equipo de investigadores.

De forma similar a la primera fase, una cuarta fase se aplicará longitudinalmente a la 
investigación, desde el comienzo y hasta el final de la misma, incorporando técnicas y actividades documentales necesarios para alcanzar los objetivos específicos.

Una quinta fase prevé la consolidación de resultados, conclusiones y recomendaciones, así como la publicación de los mismos mediante la generación de un documento, un artículo para publicación y una presentación en Power Point para dar a conocer los resultados de esta investigación en el congreso IDI UNICYT 2017, así como para otros eventos de similar naturaleza.

\section{Resultados}

Siendo esta una investigación en desarrollo, sus resultados son parciales y provienen de la ejecución parcial de las fases: primera, segunda y cuarta. Es así como se ha podido determinar que el Bitcoin es único en su creación; cualquier persona con el suficiente poder de cómputo puede crear sus propios Bitcoins mediante un proceso llamado minería. Dado que la minería requiere tanto poder de cómputo, los usuarios colaborarán entre sí y se asocian en 'pools de minería' para crear colectivamente nuevos bloques y dividir los Bitcoins recién creados (Matonis, 2013).

La seguridad de esta herramienta informática está basada en un servicio de valor agregado conocido como "Blockchain". Algunas de sus cualidades más importantes son: el hecho de Imposibilitar que un tercero determine quien, cuando y el monto de una transacción hecha por un usuario; Permite a los usuarios comprobar el pago o la identidad del pagador bajo condiciones excepcionales y Permite detener el uso de los medios de pago reportados como robados.

El Blockchain es un novedoso protocolo de criptografía, es también un esquema fuerte que aporta mucha seguridad, pero nunca será totalmente segura a pesar de sus sofisticados mecanismos para salvaguardar su integridad, basados en algoritmos llamados "agentes", dependientes de la transferencia de archivos multifuente o transferencia segmentada de archivos. Muchos expertos en finanzas y tecnología consideran al Blockchain la llave de la nueva era de expansión de Internet y que pudiera proveer la democratización de las riquezas. También hay sus detractores cuyo principal argumento es la falta de control y la posibilidad de que riqueza de dudosa procedencia pueda obtener una solución a sus problemas.

La seguridad de las criptomonedas garantiza la integridad y el equilibrio de sus estados de cuentas por medio de una estructura de agentes basada en la transferencia de archivo segmentada o transferencia de archivo multifuente, que se verifican mutuamente y que se conocen como mineros, que son, en su mayoría, usuarios que protegen activamente la red manteniendo una alta tasa de procesamiento de algoritmos.

Romper la seguridad existente en una criptomoneda es matemáticamente posible, pero el costo para lograrlo sería demasiado alto y se requeriría de una potencia de cómputo mayor que el 
Impacto de las criptomonedas en la población estudiantil universitaria de la República de Panamá

Erick Ramos, Margoth Flores y Ricardo Zeballos. (10:27)

de toda la estructura conformada por todos los mineros del sistema y, aun así, solo se tendría una probabilidad de éxito de cincuenta por ciento.

Aun cuando cada vez más se incrementa su volumen transaccional, el valor delas criptomonedas es muy volátil y se puede aseverar que no existe una guía especifica en las Normas Internacionales de Información Financiera (NIIF) sobre el tratamiento contable de las criptomonedas a pesar de lo acelerado de su uso, dejando sin respuesta la pregunta: ¿Cómo se podrán contabilizar?

Por otra parte, según las estadísticas que presenta el Instituto Nacional de Estadística y Censo (I.N.E.C.), de la Contraloría General de la República de Panamá, [14] para el año 2015 se tenían aproximadamente 129,295 estudiantes de licenciaturas en las universidades públicas y privadas de la República de Panamá. Esta cifra incluye todas las carreras que dictan la diversidad de universidades que prestan sus servicios en el territorio. También, aproximadamente el $60 \%$ de ellos estudian en los respectivos campus universitarios en la ciudad de Panamá. (I.N.E.C., 2017).

Por lo general se considera que las nuevas generaciones y en particular los jóvenes universitarios son más susceptibles de adquirir y utilizar los dispositivos o "gadgets" y las aplicaciones informáticas que permiten el uso de los nuevos procedimientos automatizados que suelen permitir mayor eficiencia y formas alternativas de hacer las cosas. Esto es especialmente cierto en aquellos aspectos lúdicos y de menor riesgo. Sin embargo, no puede darse por hecho que los jóvenes y la sociedad en general aceptaran una nueva alternativa en un aspecto que requiere tanta rigurosidad como lo son las finanzas.

En el mismo orden de ideas y de acuerdo con los datos ofrecidos por el ministerio de Educación de Panamá (MEDUCA), [15] Panamá cuenta con 29 universidades debidamente autorizadas, cinco de ellas son universidades públicas. Además, [15] se cuanta con 24 universidades particulares.

En la segunda fase se estableció hacer uso de una metodología de campo. Como instrumento para recolectar la información de este trabajo de investigación, se dispuso hacer uso de la técnica de la encuesta. El cuestionario con un total de catorce (14) preguntas, consta, de dos partes, en la primera parte se presentan cuatro (4) preguntas demográficas, en las cual se identifica género, edad, la universidad en la que estudia el estudiante encuestado y la carrera que estudia.

La segunda parte consta de diez (10) preguntas relacionadas con las variables de la investigación: reconocimiento y profundidad del mismo (ítems del 01 al 07 de la segunda parte), razones de uso de las criptomonedas (ítem 05), frecuencia con que utiliza las criptomonedas (ítem 06), interés en las criptomonedas (ítem 07), confianza en las criptomonedas (ítem 08), preferencia en alguna forma de pago (ítem 09), y percepción del futuro de las criptomonedas (ítem 10). 
El instrumento fue validado mediante la consulta a expertos en áreas relacionadas a los objetivos de la investigación, tal como la Psicología, Metodología, Economía y Ciencias Telemáticas, sus observaciones, fueron analizadas por el equipo de investigadores, y aquellas pertinentes fueron incorporadas al instrumento.

\section{Conclusiones}

Por ser una investigación en desarrollo, las conclusiones son parciales y derivan de las etapas iniciales de la misma. En tal sentido los autores consideran que ciertamente las criptomonedas son una innovación disruptiva que plantea nuevos retos a la banca internacional, a la Banca Panameña y al público en general. Queda mucha investigación por realizar.

Las criptomonedas constituyen un medio digital de intercambio de uso en varios mercados con características similares a lo que ocurre con los productos, mercancías o materias primas. llamados "Commodities" en los mercados internacionales y de forma similar a los que ocurre con el oro.

También se considera que, aunque la seguridad de las criptomonedas es buena, nunca será mayor que la de los sistemas informáticos en general, por ello se debe tener cautela en su incorporación, también, se debe hacer más investigación sobre el tema.

Más que adoptar las criptomonedas o criptovalores, se debe estudiar la tecnología de criptografía conocida como "Blockchain" para analizar los posibles usos y ventajas, así como las vulnerabilidades de tal tecnología para su uso en otras áreas del comercio de bienes y servicios.

\section{Referencias bibliográficas}

[1] Diccionario de la Lengua Española. (16-10-2017). “economía.” [En línea]. Disponible en: http://dle.rae.es/?id=ELVW605 [16-10-2017].

[2] Diccionario de la Lengua Española. (16-10-2017). “dinero.” [En línea]. Disponible en: http://dle.rae.es/?id=Doas5g0 [16-10-2017].

[3] R. Borja. (2017). "Enciclopedia de la política” [En línea]. Disponible en: http://www. enciclopediadelapolitica.org/Default.aspx $? \mathrm{i}=\mathrm{e} \&$ por $=\mathrm{d} \& \mathrm{idind}=476 \&$ termino $=[29-10-2017]$.

[4] Banco Mundial. (28-08.2014). "Los pagos digitales son esenciales para el crecimiento económico, según un informe del Banco Mundial”. [En línea]. Disponible en: http://www. bancomundial.org/es/news/press-release/2014/08/28/world-bank-report-digital-paymentseconomic-growth [16-11-2017].

[5] C.E.P.A.L. (2012). “Economía digital para el cambio estructural y la igualdad.” [En línea]. 
Impacto de las criptomonedas en la población estudiantil universitaria de la República de Panamá Erick Ramos, Margoth Flores y Ricardo Zeballos. (10:27)

Disponible en: https://www.cepal.org/ilpes/noticias/paginas/3/54303/economia_digital_para_ cambio.pdf [27-10-2017].

[6] Órgano Judicial del Gobierno de Panamá. (24-07-2008).“Gaceta Oficial Digital 26090, del 24 de julio de 2008”. [En línea]. Disponible en: http://www.organojudicial.gob.pa/wp-content/ uploads/2016/11/ Ley-51-de-2008.pdf [20-11-2017].

[7] J. Matonis. (2013). "Bitcoin 101 How to get started with the new trend in virtual currencies." [En línea]. Disponible en: https://www.coursehero.com/file/10757229/ bitcoin-101/ [27-10-2017].

[8] Robert Mueller III. (03-2012). "RSA Cyber Security Conference San Francisco, CA". [En línea]. Disponible en: https://archives.fbi.gov/archives/news/speeches/ combating-threats-inthe-cyber-world-outsmarting-terrorists-hackers-and-spies\#disablemobile [23-11-2017].

[9] J. Surowiecki. (23-08-2011). "Cryptocurrency”. MIT Technology Review. [En línea]. Disponible en: https://www.technologyreview.com/s/425142/cryptocurrency/ [29-10-2017].

[10] O. Castaño. (10-10-2017). "BITCÓIN EN VEZ DE DÓLAR.” [En línea]. Disponible en: http://www.martesfinanciero.com/history/2017/10/10/dossier3.asp [17-10-2017].

[11] J. Becerra, citada por: O. Castaño. (10-10-2017). "BITCÓIN EN VEZ DE DÓLAR." [En línea]. Disponible en: http://www.martesfinanciero.com/history/2017/10/10/dossier3.asp [1710-2017].

[12] Diario la Estrella de Panamá. (10-05-2015) "El sistema bancario panameño: un gigante en plena maduración. “ [En línea]. pp. Portada. Disponible en: http:/laestrella.com.pa/panama/ nacional/sistema-bancario-panameno-gigante-plena-maduracion/23864583 [15-10-2017].

[13] Diario La Estrella de Panamá. (06-05-2015) "Evolución y competitividad del sector bancario panameño." [En línea]. pp. Portada. Disponible en: http:/laestrella.com. pa/economia/ evolucion-competitividad-sector-bancario-panameno/23863569 [15-10-2017].

[14] El Instituto Nacional de Estadística y Censo (INEC) de la Contraloría General de la República de Panamá. (2015). "El Instituto Nacional de Estadística y Censo (INEC) presenta en esta publicación, los datos correspondientes a las estadísticas educativas del año escolar 2015". [En línea].Disponible en: https://www.contraloria.gob.pa/inec/Publicaciones/Publicaciones.aspx ?ID_SUBCATEGORIA=41\&ID_PUBLICACION $=835 \&$ ID_IDIOMA $=1 \&$ ID_CATEGORIA $=6$ [26-11-2017]. 
[15] Comisión Técnica de Fiscalización. (2012) "Boletín Informativo enero a junio de 2012" [En línea]. Disponible en: http://www.ctf.ac.pa/boletininformativo/univ_que_ingresaron_pros_eva _acre_univ.htm [20-11-2017].

[16] Diario La Prensa. (15-10-2017).“Panamá es uno de los países de la región que menos presupuesto destina para el desarrollo de la ciencia, tecnología e innovación en Latinoamérica.“[Edición impresa]. pp. Portada. [ 15-10-2017].

[17] Diario Página Siete. (21-06-2014). "BCB prohíbe uso de moneda virtual conocida como Bitcoin. "[En línea]. Disponible en: http://www.paginasiete.bo/ economia/2014/6/21/prohibemoneda-virtual-conocida-como-bitcoin-24782.html [16-10-2017].

[18] Don Tapscott, (06-2016). "Cómo la cadena de bloques está cambiando los negocios." [En línea].Disponible en: www.ted.com/talks/don_tapscott_how_the_blockchain_is_changing _ money_and_business?language $=$ es\#t-391652 [01-12-2017].

[19] Usman W. Chohan. (26-08-2017). "Cryptocurrencies: A Brief Thematic Review." [En línea]. Disponible en: https://papers.ssrn.com/sol3/papers.cfm?abstract_id=3024330[01-12-2017].

[20] BBC.News. (02-05-2016) "Australian Craig Wright claims to be Bitcoin creator ." [En línea]. Disponible en: http://www.bbc.com/news/technology-36168863 [01-12-2017].

[21] Infotechnology.com. (07-12-2017). "UNA DE LAS MÁS GRANDES EMPRESAS DE BITCOIN FUE HACKEADA, ¿POR SU PROPIO CEO?” [En línea]. Disponible en: http://www. infotechnology.com/negocios/Una-de-las-mas-grandes-empresas-de-Bitcoin-fue-hackeada-porsu-propio-CEO-20171207-0001.html [08-12-2017]. 